\title{
Correction to: Hepatic metastatic paraganglioma 12 years after retroperitoneal paraganglioma resection: a case report
}

Zhao-Ru Dong ${ }^{1}$, Yan-Ni Xia ${ }^{2}$, Yue-Yi Zhao ${ }^{3}$, Rui Wu ${ }^{1}$, Kai-Xuan Liu' ${ }^{1}$ Kai Shi ${ }^{1}$, Lun-Jie Yan ${ }^{1}$, Cheng-Yu Yao', Yu-Chuan Yan ${ }^{1}$ and Tao Li'

\section{Correction to: BMC Gastroenterology}

$$
\text { https://doi.org/10.1186/s12876-019-1061-6 }
$$

Following publication of the original article [1], the author reported that their family name was misspelled. The details are as follows:

Incorrect name in the original article:

Rui-Yi Zhao.

Correct name:

Yue-Yi Zhao.

The original article has been corrected.

\section{Author details}

'Department of general surgery, Qilu Hospital, Shandong University, Jinan 250012, People's Republic of China. ${ }^{2}$ Department of Operating Room, Qilu Hospital, Shandong University, Jinan 250012, China. ${ }^{3}$ Department of Clinical Laboratory, Shandong First Medical University, Jinan 250012, China.

Published online: 26 August 2019

\section{Reference}

1. Dong ZR, et al. Hepatic metastatic paraganglioma 12 years after

retroperitoneal paraganglioma resection: a case report. BMC

Gastroenterology. 2019;19:142. https://doi.org/10.1186/s12876-019-1061-6.

\footnotetext{
*Correspondence: litao7706@163.com

1 Department of general surgery, Qilu Hospital, Shandong University, Jinan

250012, People's Republic of China

Full list of author information is available at the end of the article
} 